\title{
Linx
}

Revue des linguistes de l'université Paris X Nanterre

$7 \mid 1995$

Saussure aujourd'hui

\section{Le « point de vue » dans les sciences}

\section{Luis J. Prieto}

\section{OpenEdition}

\section{Journals}

Édition électronique

URL : http://journals.openedition.org/linx/1223

DOI : 10.4000/linx.1223

ISSN : 2118-9692

\section{Éditeur}

Presses universitaires de Paris Nanterre

\section{Édition imprimée}

Date de publication : 1 septembre 1995

Pagination : 387-393

ISSN : 0246-8743

\section{Référence électronique}

Luis J. Prieto, « Le « point de vue » dans les sciences », Linx [En ligne], 7| 1995, mis en ligne le 23 juillet 2012, consulté le 20 avril 2019. URL : http://journals.openedition.org/linx/1223; DOI : 10.4000/

linx.1223

Ce document a été généré automatiquement le 20 avril 2019.

Département de Sciences du langage, Université Paris Ouest 


\title{
Le « point de vue » dans les sciences
}

\author{
Luis J. Prieto
}

1 Vue la diversité de ce qu'on désigne du nom de "sémiologie" je crois nécessaire de commencer mon exposé en précisant brièvement en quoi consiste la discipline que j'appelle ainsi et dans laquelle se placent mes recherches D'une certaine façon cette sémiologie est une théorie de la connaissance, une théorie de la connaissance qui ne fait cependant pas double emploi avec celle des philosophes dans la mesure où elle tient compte du sujet - ce qu'à mon avis omettent de faire les philosophes - en explicitant la raison d'être des connaissances c'est-à-dire les raisons qui amènent le sujet à les construire et s'en servir.

2 La raison d'être d'une connaissance se trouve bien entendu pertinence. Or le principe de base de la discipline à laquelle je me reférerai désormais avec le nom de "sémiologie" consiste justement à affirmer que la validité d'une connaissance ne dépend pas seulement du critère traditionnellement admis de la vérité mais aussi d'un autre critère, de surcroît logiquement prioritaire, qui est celui de la pertinence. La priorité de ce dernier critère à l'égard de la vérité se manifeste dans le fait que le sujet s'intéresse à la vérité ou à la fausseté d'une connaissance seulement si cette connaissance est pour lui pertinente. La vérité d'une connaissance est un rapport entre celle-ci et son objet, et ce rapport ne dépend pour rien du sujet ni par conséquent du contexte socioculturel dans lequel le sujet construit les connaissances. La pertinence est par contre un rapport entre la connaissance et le sujet, c'est-à-dire qu'elle dépend en définitive des besoins et des désirs socialement conditionnés du sujet: la pertinence est de ce fait la composante historique et sociale de toute connaissance réelle, c'est-à-dire, de toute connaissance dont des sujets se servent ou se sont servis effectivement.

Le principe de pertinence permet de résoudre un vieux problème qui se pose à l'épistémologie ou en tout cas aux épistémologies d'inspiration marxiste, le problème de pouvoir affirmer le caractère historique de toute connaissance tout en évitant de tomber dans l'idéalisme c'est-à-dire dans la négation de l'existence objective de la réalité. La garantie de l'existence objective de la réalité ou, pour le dire en d'autres termes, de l'existence de la réalité indépendante $d u$ fait que l'on construise ou non des connaissances d'elle et des connaissances qu'on en construit éventuellement, nous est 
donnée par le principe de non contradiction, qui a trait à la vérité. On peut construire du même objet plusieurs connaissances vraies, voire une infinité : la connaissance qu'une caissière de supermarché a d'une pièce de monnaie n'est par exemple forcément pas la même connaissance qu'en a un numismate et cependant ces connaissances peuvent être toutes les deux des connaissances vraies. Mais on ne peut pas construire d'un même objet deux connaissances contradictoires entre elles qui seraient en même temps l'une et l'autre vraies. C'est cette limite que la réalité impose aux connaissances qu'on peut construire d'elle qui nous assure que la réalité possède une existence indépendante de ces connaissances. Cependant, si pour affirmer le caractère socioculturel des connaissances on relativise leur vérité, si l'on dit qu'une connaissance, vraie dans un contexte socioculturel, peut être fausse dans un autre on élimine le principe de non contradiction et en l'éliminant on nie l'existence objective de la réalité.

4 Les épistémologues, du moins ceux qui s'inspirent de la philosophie matérialiste, ne sont certes pas tombés dans ce piège. Ils ont essayé de concilier le caractère socioculturel de la connaissance et son objectivité en ayant recours au principe du "rapprochement perpétuel". Ils partent de l'idée, fausse mais qu'ils ne sont nullement seuls à soutenir ${ }^{1}$, que la connaissance parfaite serait celle qui reflète l'objet "tel qu'il est en réalité », l'objet dans la totalité de ses déterminations ${ }^{2}$. Le caractère socioculturel des connaissances, expliquent-ils, résulte du fait que les différents groupes sociaux se sont diversement rapprochés de cette connaissance "parfaite" elle-même inaccessible à l'intelligence limitée de l'homme mais dont l'intelligence de l'homme est en quête perpétuelle. Le caractère socioculturel de la connaissance serait ainsi un fait purement négatif : c'est parce que l'homme n'est pas capable de construire la connaissance "parfaite" d'un objet que la connaissance qu'il en construit est socioculturelle. Si l'intelligence de l'homme était assez puissante pour construire une telle connaissance elle serait a-historique puisqu'elle serait la même pour tous: il est impossible en effet de construire deux connaissances qui tiendraient toutes deux compte de la totalité des déterminations de l'objet et qui ne seraient pas toutes deux la même connaissance.

5 Avec le principe de pertinence le caractère socioculturel apparait au contraire comme une composante positive de toute connaissance. Le fait qu'une connaissance reflète toujours partiellement l'objet ne constitue nullement une tare consécutive à la limitation de l'intelligence qui l'a construite: la connaissance parfaite n'est pas celle qui reflète l'objet "tel qu'il est" mais celle qui retient de l'objet, tout ce qui compte, et seul ce qui compte pour l'intérêt qui amène le sujet à s'occuper de cet objet - intérêt conditionné bien entendu par le contexte social et culturel dans lequel le sujet se trouve nécessairement. La connaissance parfaite est, en d'autres termes, celle qui tient compte dans l'objet de tout ce qui est pertinent et seulement de ce qui est pertinent pour le sujet: une caissière de supermarché qui tiendrait compte par exemple de l'année de frappe de chacune des pièces de monnaie qu'elle reçoit ne connaîtrait certainement pas "mieux" ces pièces qu'une collègue qui n'en tiendrait pas compte, mais cela, qui est valable pour la caissière, ne l'est très probablement pas pour le numismate.

6 En disant cela personne n'entend bien entendu nier la limitation de l'intelligence humaine ni sa difficulté à atteindre la connaissance parfaite même dans le sens qu'on vient de définir. On peut même admettre l'impossibilité de l'homme de parvenir à une telle connaissance et donc le perpétuel rapprochement dans lequel l'homme se trouve à son égard. Ce n'est cependant pas de cela - fait négatif - ou du moins pas seulement de cela que dépend le caractère socioculturel de la connaissance mais bien du fait - positif - 
qu'elle est en tout cas pertinente : même si l'on réussissait à construire la connaissance "parfaite" dans le sens courant c'est-à-dire la connaissance d'un objet qui tiendrait compte de la totalité des déterminations de celui-ci une telle connaissance serait socioculturelle du moment que si on la construit c'est parce que l'on considère que toutes les déterminations de l'objet sont pertinentes et cette pertinence ne provient pas de l'objet mais du sujet socio-culturellement conditionné.

7 L'intérêt qui amène le sujet à s'occuper d'un objet, intérêt dont dépend ce qui dans l'objet est pour lui pertinent et qui va de ce fait être retenu dans la connaissance qu'il en construit, n'est rien d'autre que le point de vue saussurien. I1 me semble nécessaire de préciser, pour éviter certaines erreurs, que le point de vue saussurien est un point de vue relationnel, qu'il faut distinguer des points de vue inhérents desquels on peut également considérer un objet. Considérer un objet du point de vue de sa couleur, par exemple, ou de sa forme, de son poids, etc. c'est le considérer d'un point de vue inhérent. Le point de vue dont parle Saussure, duquel va dépendre la pertinence de la connaissance, est celui d'une relation dans laquelle l'objet en question se trouve à l'égard d'un autre objet, relation qui, à mon avis, est en définitive soit celle de causalité soit celle de signification: on cherche a établir non la totalité des caractéristiques d'un objet mais celles dont on considère (à tort ou à raison) qu'il doit être pourvu soit pour pouvoir être la cause d'un objet matériel déterminé soit pour signifier un objet mental déterminé. En disant "objet matériel" et "objet mental" déterminés j'entends bien entendu me référer à un objet matériel ou à un objet mental pourvus d'une certaine identité, laquelle apparaît à son tour au sujet comme pertinente parce qu'il considère l'objet matériel ou l'objet mental en question d'un point de vue également relationnel. C'est par ailleurs du point de vue relationnel, c'est-à-dire saussurien, duquel le sujet considère l'objet que dépendent les points de vue inhérents qui sont pour lui pertinents. Ainsi c'est parce que je considère par exemple les bâtons de craie que je trouve sur la table de la salle de cours du point de vue de leur relation avec les objets graphiques que je peux produire par leur moyen sur le tableau noir que je les considère du point de vue inhérent de la couleur (par exemple, bleue ou blanche) et non pas du point de vue également inhérent de la forme (par exemple cylindrique ou prismatique).

8 Lorsque la phonologie pragoise, dont s'inspire ce que je dis ci-dessus, établit, se fondant sur un célèbre passage du Cours de Saussure ${ }^{3}$, que l'identité que le sujet parlant reconnaît aux sons, c'est-à-dire la façon pour lui pertinente de les connaître, résulte de leur relation aux signifiés, certains linguistes ont cru y voir l'extension aux sciences de l'homme de quelque chose qui aurait été depuis toujours admis pour ce qui concerne les sciences de la nature : chaque science de la nature choisirait un point de vue duquel elle aborde l'étude de la réalité et ce serait ce point de vue qui la définit. De façon analogue, la phonologie pragoise « choisirait » le rapport aux signifiés comme point de vue duquel elle aborderait l'étude des sons ${ }^{4}$.

9 Cette façon de voir les choses est peut être trop simpliste en ce qui concerne les sciences de la nature mais elle est, toujours en ce qui concerne les sciences de la nature, fondamentalement vraie pour le problème qui nous intéresse: un biochimiste, par exemple, qui examine un échantillon de cellules d'un patient considère ces cellules seulement de certains points de vue inhérents, ceux qu'il considère pertinents d'un point de vue relationnel, celui du rapport dans lequel ces cellules se trouvent à l'égard d'un processus pathologique qui pourrait se développer dans l'organisme du patient. L'assimilation, au contraire, de la démarche phonologique à celle des sciences de la 
nature est totalement fausse. A propos du biochimiste, par exemple, on peut dire qu'il choisit le point de vue relationnel duquel il considère les cellules parce que, quelles que soient les contraintes socioculturelles auxquelles il est soumis, c'est en définitive luimême qui aborde l'objet de ce point de vue. Le phonologue par contre n'étudie pas les sons et à plus forte raison ne choisit pas le point de vue relationnel duquel il les considère. C'est le sujet parlant qui considère les sons d'un point de vue relationnel, celui de leur relation aux signifiés, ce qui l'amène à ne tenir compte dans les sons que des caractéristiques et donc des points de vue inhérents qu'il considère pertinents de ce point de vue relationnel. Ensuite c'est cette façon dont le sujet parlant identifie les sons c'est-à-dire la façon dont il les connait qui constitue l'objet de la phonologie. L'objet du phonologue est donc une connaissance qu'il trouve déjà construite par le sujet parlant, et sa tâche, dont il s'est acquitté en s'inspirant de Saussure, est celle d'expliciter le point de vue relationnel dont résulte la pertinence que cette connaissance des sons possède pour le sujet parlant.

Or il me semble que la phonologie constitue un cas paradigmatique des disciplines qu'on appelle les "sciences de l'homme"5 : face aux sciences de la nature, qui étudient la réalité matérielle, les sciences de l'homme étudient les connaissances, scientifiques ou non, de la réalité matérielle en expliquant leur raison d'être grâce à la mise en lumière du point de vue qui les rend pertinentes pour le sujet qui les construit ou qui s'en sert. La tâche des sciences de l'homme consisterait donc à expliciter la composante socioculturelle que comporte toute connaissance réelle et qui la rend un objet historique. L'archéologue, par exemple, lorsque face à un ustensile de l'âge de pierre il se demande "qu'est-ce que c'est ?", ne se demande pas ce que l'objet en question est de son propre point de vue (par exemple, d'un point de vue inhérent, un bloc de silex ou, d'un point de vue relationnel, un joli presse-papiers, etc.) mais ce que l'objet en question a été pour celui qui l'a produit ou pour celui qui l'a utilisé. L'archéologue se demande en d'autres termes à quoi l'objet en question servait, c'est-à-dire, quel était le point de vue relationnel duquel son producteur et son utilisateur le considéraient et duquel il peut déduire la façon dont ces derniers le connaissaient. C'est pourquoi toutes les sciences de l'homme et seules les sciences de l'homme sont exposées à l'ethnocentrisme: on a affaire à l'ethnocentrisme chaque fois que le chercheur oublie son rôle, qui est celui d'expliciter le point de vue sur lequel se fonde, ou s'est fondé, un autre sujet pour construire la connaissance d'un objet et substitue à ce point de vue le sien propre.

\section{NOTES}

1. On la trouve dans des linguistes comme A. Martinet, « Où en est la phonologie ? ", Lingua vol. I, s.d., P. 37.

2. Je considère qu'il est absurde de parler d'une connaissance qui tiendrait compte de X la totalité des caractéristiques d'un objet, c'est-à-dire qui l'épuiserait intentionnellement, du fait déjà que même si on parvenait à une telle totalité on ne serait pas en mesure de se rendre compte qu'on y est parvenu. Ici, cependant, je laisse de côté ce problème. Remarquons d'ailleurs que la connaissance d'un objet qui le refléterait « tel qu'il est » serait tout aussi inutile que l'est la carte 
de l'empire aussi grande que l'empire dont parle J. L. Borges dans un de ses petits récits, « De la rigueur de la science », Histoire de l'infamie, Histoire de l'éternité, coll. 10/18, p. 129 et ss.

3. "Une suite de sons n'est linguistique que si elle est le support d'une idée », Cours de linguistique générale, 4ème éd., Paris, Payot, 1949" p. 144.

4. C'est ce que pense par exemple A. Martinet : v. « Où en est la phonologie ? », Lingua, vol I, s.d., p. 37 et « Some basic principles of functional linguistics», La Linguistique, vol. XIII, 1977, p. 9.

5. Traditionnellement on ne dit pas « sciences de l'homme » mais « sciences humaines ». Ce n'est pas sans une raison précise que je m'écarte de cette tradition. Le rapport de l'adjectif avec le nom me semble en effet beaucoup plus étroit que celui du génitif. Or, toutes les sciences (ainsi bien que toutes les connaissances non scientifiques) sont foncièrement humaines du fait qu'elles possèdent toujours une pertinence qui constitue même leur raison d'être et que cette pertinence ne vient pas de l'objet mais du sujet soumis à un contexte social et culturel. Ensuite, selon son objet, une science, nécessairement humaine, peut être soit une science de la nature soit une science de l'homme. Surtout l'expression «sciences naturelles" me semble particulièrement choquante et susceptible de renforcer des positions épistémologiques idéologiquement dangereuses. 